\title{
Dynamical Generation of Noiseless Quantum Subsystems
}

\author{
Lorenza Viola ${ }^{1}$, Emanuel Knill ${ }^{2}$, and Seth Lloyd ${ }^{1 \dagger}$ \\ 1 d'Arbeloff Laboratory for Information Systems and Technology, Department of Mechanical Engineering, \\ Massachusetts Institute of Technology, Cambridge, Massachusetts 02139 \\ ${ }^{2}$ Los Alamos National Laboratory, Los Alamos, New Mexico 87545
}

\begin{abstract}
We present control schemes for open quantum systems that combine decoupling and universal control methods with coding procedures. By exploiting a general algebraic approach, we show how appropriate encodings of quantum states result in obtaining universal control over dynamicallygenerated noise-protected subsystems with limited control resources. In particular, we provide an efficient scheme for performing universal encoded quantum computation in a wide class of systems subjected to linear non-Markovian quantum noise and supporting Heisenberg-type internal Hamiltonians.
\end{abstract}

03.67.-a,03.67.Lx,03.65.-w,89.70.+c

Quantum bang-bang control has recently emerged as a general strategy for manipulating quantum evolutions by enforcing suitable time scale separations between the controller and the natural dynamics of the system [i] For open quantum systems, this has lead to establishing quantum error suppression schemes, whereby active decoupling from environmental noise is achieved by continuously undoing system-bath correlations on time scales that are short compared to the typical memory time of the bath [2]. Decoupling techniques were shown to be consistent with efficient quantum information processing [3], thereby offering an alternative scenario compared to error-correcting [4] and error-avoiding quantum codes [5]. In contrast to the latter methods, no redundant encoding is necessary for preserving or manipulating quantum information provided that the required control operations can be implemented. However, one may ask whether quantum coding could be advantageous or necessary in situations where the available control options are limited.

Answering the above question naturally connects the decoupling formalism with the notion of noiseless subsystem that has been identified as the most general route to noise-free information storage [6]. The basic philosophy is to envision the bang-bang control procedure as a tool for effectively endowing the system dynamics with a nontrivial group of symmetries. Such symmetries generate structures in the system's state space which are in principle inaccessible to unwanted interactions and are therefore suited for encoding quantum information. Mathematically, the crucial requirement relates to the reducibility properties of operator algebras associated with the action of the decoupling group. Variants of the same basic idea have been argued to lie at the heart of all existing approaches for stabilizing quantum information in a recent work by Zanardi [7].

In this Letter we examine the implications of the above concept within the decoupling framework, by showing that the action of the control group allows for a complete classification of the choices available for both safe information encoding and universal control over coded states. At variance with the case where noiseless subsys- tems emerge by virtue of preexisting static symmetries in the overall Hamiltonian, the dynamical origin of the noise-protected structures also precisely constrains the admissible methods for implementing universal control in a way which simultaneously preserves the effect of decoupling as well as the selected coding space.

Using coding methods has several attractive consequences. First, bang-bang operations are needed only for noise suppression. Additional manipulations on encoded subsystems become fully implementable via weak strength controls [3]. Second, for schemes where the relevant Hamiltonians are allowed to be turned on or off slowly, an advantage is that the corresponding pulses can be made more easily frequency-selective. Finally, coded states may be intrinsically more robust against imperfections in the decoupler operations. For a potentially large class of quantum information processors characterized by linear quantum noise, we outline a scheme where noisedecoupling involves a minimal set of two collective bangbang rotations and universal quantum computation on encoded qubits can be performed entirely through slow tuning of two-body bilinear interactions.

Decoupling.- Let $S$ be a finite-dimensional quantum system with self-Hamiltonian $H_{S}$ on $\mathcal{H}_{S}$, $\operatorname{dim}\left(\mathcal{H}_{S}\right)=d$. $S$ interacts with the environment $B$ via a Hamiltonian $H_{S B}=\sum_{\alpha} E_{\alpha} \otimes B_{\alpha}$, the $B_{\alpha}$ 's being linearly independent environment operators. The error operators $E_{\alpha}$ are assumed to belong to a linear space $\mathcal{E}$ that we call the interaction space. We require that $\operatorname{tr}\left(E_{\alpha}\right)=0$, thereby removing from $H_{S B}$ the internal evolution of the environment. Let $\mathcal{A}_{\mathcal{E}}$ denote the algebra generated by the identity, $H_{S}$, and $\mathcal{E}$. $\mathcal{A}_{\mathcal{E}}$ is a subalgebra of the full operator algebra $\operatorname{End}\left(\mathcal{H}_{S}\right)$ closed under Hermitian transpose $\left({ }^{\dagger}\right.$-closed). For $n$-qubit systems, $\mathcal{H}_{S} \simeq \mathbb{C}^{d}$, $\operatorname{End}\left(\mathcal{H}_{S}\right) \simeq \operatorname{Mat}(d \times d, \mathbb{C})$, with $d=2^{n}$.

In its essence, decoupling via bang-bang (b.b.) control relies on the idea of exploiting full strength/fast switching control actions [1], meaning that a certain set of Hamiltonians can be (ideally) turned on/off instantaneously with arbitrarily large strength. Let $\mathcal{G}$ denote a finite group determining the realizable b.b. operations 
(decoupling group), $|\mathcal{G}|=\operatorname{order}(\mathcal{G})$. We identify the abstract group $\mathcal{G}$ with its image under a unitary, faithful representation $\mu$ by $d \times d$ matrices. A decoupler operates by subjecting the overall system to a cyclical time evolution, the elementary temporal loop (of duration $T_{c}$ $=$ cycle time) being designed to effect a suitable grouptheoretical averaging determined by $\mathcal{G}$. In the ideal limit of arbitrarily fast cycle time, the action of the decoupler is equivalent to a modification of the effective dynamics according to $\mathcal{A}_{\mathcal{E}} \mapsto \Pi_{\mathcal{G}}\left(\mathcal{A}_{\mathcal{E}}\right), \Pi_{\mathcal{G}}$ being defined by [2, 3, 8] 8 ]

$$
X \mapsto \Pi_{\mathcal{G}}(X)=\frac{1}{|\mathcal{G}|} \sum_{g \in \mathcal{G}} g^{\dagger} X g, \quad X \in \mathcal{A}_{\mathcal{E}} .
$$

The quantum operation $\Pi_{\mathcal{G}}$ is identical with the projector on the commutant (or centralizer) of $\mathcal{G}$ in $\operatorname{End}\left(\mathcal{H}_{S}\right)$, $Z(\mathcal{G})=\left\{\mathcal{O} \in \operatorname{End}\left(\mathcal{H}_{S}\right) \mid[\mathcal{O}, g]=0 \forall g \in \mathcal{G}\right\}$. Since $\Pi_{\mathcal{G}}\left(H_{S}\right)=H_{\text {eff }} \in Z(\mathcal{G})$, the decoupler essentially induces a $\mathcal{G}$-symmetrization of the dynamics due to $H_{S}$.

The commutant $Z(\mathcal{G})$ has a natural structure as a subalgebra of $\operatorname{End}\left(\mathcal{H}_{S}\right)$. A second algebraic structure associated with $\mathcal{G}$ is the algebra generated by $\mathcal{G}, \mathbb{C G}$, which is the (at most) $|\mathcal{G}|$-dimensional vector space spanned by complex combinations of elements in $\mathcal{G}[9]$. Let $\mathbb{C G}^{\prime}$ denote the set of operators commuting with $\mathbb{C G}$. Clearly, $\mathbb{C G}^{\prime}=Z(\mathcal{G})$. The fact that both $\mathbb{C} \mathcal{G}$ and $\mathbb{C G}^{\prime}$ are ${ }^{\dagger}$ closed subalgebras of $\operatorname{End}\left(\mathcal{H}_{S}\right)$ will play an important role. $\mathbb{C G}$ and $\mathbb{C} \mathcal{G}^{\prime}$ are linked together by the property of reducibility [9]. $\mathbb{C G}$ is said to be irreducible (and $\mathcal{G}$ to act irreducibly on $\mathcal{H}_{S}$ ) if $\mathbb{C G}^{\prime}=\{\lambda \cdot \mathbb{1}\}=\mathbb{C} \mathbb{1}$. A similar definition applies to $\mathbb{C} \mathcal{G}^{\prime}$. Since $\mathbb{C} \mathcal{G}^{\prime \prime}=\mathbb{C G}$, the non-triviality of $\mathcal{G}$ automatically implies that $\mathbb{C G}^{\prime}$ is reducible. Whether or not $\mathbb{C} \mathcal{G}$ acts irreducibly on $\mathcal{H}_{S}$ distinguishes, at the algebraic level, between maximal decoupling, where $\mathbb{C} \mathcal{G}^{\prime}=\mathbb{C} \mathbb{1}$, and selective decoupling, in which case $\mathbb{C G}^{\prime} \neq \mathbb{C} \mathbb{1}$ [2].

The goal of decoupling is to dynamically maintain evolutions of the system so as to have a place where quantum information can safely reside and undergo the required logical manipulations. The possibility to carry out such a program without resorting to redundant encoding was demonstrated in [3]. Is this the only relevant situation?

Encoding.- The basic idea is provided by the notion of a subsystem [6]. Mathematically, subsystems are identified as factors of subspaces by observing that the action of $\mathbb{C G}$ and $\mathbb{C G}^{\prime}$ on $\mathcal{H}_{S}$ can be represented as [6, [6]

$$
\begin{aligned}
\mathbb{C G} & \simeq \oplus_{J} \mathbb{1}_{n_{J}} \otimes \operatorname{Mat}\left(d_{J} \times d_{J}, \mathbb{C}\right), \\
\mathbb{C G}^{\prime} & \simeq \oplus_{J} \operatorname{Mat}\left(n_{J} \times n_{J}, \mathbb{C}\right) \otimes \mathbb{1}_{d_{J}},
\end{aligned}
$$

where the index $J$ labels the $J$-th $d_{J}$-dimensional irreducible component of $\mathbb{C G}$, appearing with multiplicity $n_{J}$. Obviously, $\sum_{J} n_{J} d_{J}=d$. Such representations are associated with the following decomposition of $\mathcal{H}_{S}$ :

$$
\mathcal{H}_{S} \simeq \oplus_{J} \mathcal{H}_{J} \simeq \oplus_{J} \mathcal{C}_{J} \otimes \mathcal{D}_{J},
$$

with $\operatorname{dim}\left(\mathcal{C}_{J}\right)=n_{J}, \operatorname{dim}\left(\mathcal{D}_{J}\right)=d_{J}$. Results (2)-(4) stem from the general decomposition theory of ${ }^{\dagger}$-closed operator algebras. As argued in [6,7], they provide the common algebraic ground for discussing noise control strategies. In our setting, the above relationships are linked to the decomposition of $\mu$ according to the irreducible representations (irreps) of $\mathcal{G}, \mu=\oplus_{J} n_{J} \mu_{J}$ [9]. Eq. (4i) reflects the fact that the subspace $\mathcal{H}_{J}$ of states transforming according to $\mu_{J}$ arises from $n_{J}$ replicas of a $d_{J}$-dimensional irrep. In a suitably chosen orthonormal basis of $\mathcal{H}_{J}$, $\left\{|J, l, m\rangle \mid l=1, \ldots, n_{J} ; m=1, \ldots, d_{J}\right\}$, such a one-toone mapping is given by a correspondence of the form $|l, m\rangle \leftrightarrow|l\rangle \otimes|m\rangle$. Thus, the $J$-th eigenspace factorizes into the tensor product of two factors $\mathcal{C}_{J}$ and $\mathcal{D}_{J}$, carrying irreps of $\mathbb{C} \mathcal{G}^{\prime}$ and $\mathbb{C G}$ respectively. By construction, the dimensions of $\mathbb{C \mathcal { G } ^ { \prime }}$-irreps are found as multiplicities of $\mathbb{C} \mathcal{G}$-irreps, and vice versa.

The physical meaning behind the above construction is simple: The overall state space $\mathcal{H}_{S}$ is decomposed into invariant subspaces $\mathcal{H}_{J}$, each of which can be regarded as the state space of a bipartite system. For fixed $J, \mathcal{C}_{J}$ is the state space of a subsystem which is only acted on non-trivially by operators in $\mathbb{C} \mathcal{G}^{\prime}$, while $\mathcal{D}_{J}$ is the state space of a subsystem which is only acted on non-trivially by operators in $\mathbb{C G}$. Clearly, one is left with the freedom of exploiting any of those subsystems for encoding quantum states. Under what conditions is such an encoding noiseless?

Let us first consider encoding in the left factors $\mathcal{C}_{J}$ ("commutant coordinates"), assuming that $n_{J}>1$. When, in situations with underlying static symmetry, the decomposition (2) is applied to the interaction algebra $\mathcal{A}_{\mathcal{E}}$, this generalizes the standard case of noiseless subspaces, where coding takes place in the singlet sector of $\mathcal{A}_{\mathcal{E}}, d_{J_{0}}=1, \mathcal{C}_{J_{0}} \simeq \mathcal{H}_{J_{0}}$ 河, 10. Within the decoupling framework, protection against environmental noise is guaranteed if $\Pi_{\mathcal{G}}(\mathcal{E})=0$ i.e., $\mathcal{E}$ is correctable by $\mathcal{G}$ [2, 3]. In fact, this condition is no longer necessary and can be replaced by the weaker requirement $\Pi_{\mathcal{G}}(\mathcal{E}) \in \mathbb{C} \mathcal{G}^{\prime} \cap \mathbb{C G}$, meaning that the effective error space belongs to the so-called center of $\mathbb{C G}$. Noise suppression is then ensured by the trivial action of the central elements on $\mathcal{C}_{J}, \mathbb{C G}^{\prime} \cap \mathbb{C G} \simeq \oplus_{J} q_{J} \mathbb{1}_{n_{J}} \otimes \mathbb{1}_{d_{J}}, q_{J} \in \mathbb{C}$. Note that $\mathbb{C G}^{\prime} \cap \mathbb{C G}=\mathbb{C G}$ for Abelian decouplers.

As a second coding method, we can choose the right factors $\mathcal{D}_{J}$ ("group coordinates"). Such an option requires $d_{J}>1$, thereby excluding one-dimensional irreps. As a limiting case, this is the only possibility if $\mathcal{G}$ acts irreducibly on $\mathcal{H}_{S}$, in which case the decomposition (2) collapses to a single term $\mathbb{C G} \simeq \operatorname{Mat}(d \times d, \mathbb{C})$ and the whole space is a noiseless subsystem [7]. In general, since symmetrized noise generators $\Pi_{\mathcal{G}}\left(E_{\alpha}\right) \in \mathbb{C G}^{\prime}$ act trivially on factors carrying a $\mathbb{C} \mathcal{G}$-irrep, subsystems of the form $\mathcal{D}_{J}$ are automatically immune to environmental noise irrespective of the decoupler's ability to suppress the errors. Although the overall effective dynamics is not unitary in this case, corruption of states in $\mathcal{D}_{J}$ is fully prevented due to their symmetry.

In addition to protecting against the environment, encoding may also offer improved stability against faults 
in the implementations of b.b. control. In particular, while imperfections of operations in $\mathcal{G}$ directly affect the group component, states that carry $\mathbb{C G}^{\prime}$-coordinates are still unaffected as long as $\mathbb{C G}^{\prime}$ is preserved. Thus, encoding in the commutant degrees of freedom $\mathcal{C}_{J}$ is robust against imperfections of the b.b. rotations which stay in $\mathbb{C G}$. Experience from nuclear magnetic resonance suggests that such imperfections do not severely affect the ability of the decoupler to maintain noiselessness of the commutant degrees of freedom. This effect will be analyzed elsewhere.

Universal control.- Since the group-theoretic averaging of the decoupler is intrinsically associated with a minimum time scale $T_{c}$ [2,3], it is not surprising that control operations are to be effected according to different timing criteria depending on whether the intended action is on the group or the commutant coordinates. Regardless of the choice of $\mathcal{C}_{J}$ or $\mathcal{D}_{J}$ as the preferred coding space, transformations over a given subsystem should not be allowed to ever draw states out of the protected factor. This determines the symmetry of the Hamiltonians to be applied for control, $H \in \mathbb{C G}^{\prime}$ or $H \in \mathbb{C} \mathcal{G}$ for action on $\mathcal{C}_{J^{-}}$ or $\mathcal{D}_{J}$-subsystems respectively. Since the application of Hamiltonians in $\mathbb{C G}^{\prime}$ does not interfere with the decoupler performances, encoding in $\mathcal{C}_{J}$ has the virtue that programming operations can be effected via the weak strength/slow switching scheme introduced in [3]. On the other hand, when encoding in $\mathcal{D}_{J}$ is chosen, slow application of arbitrary Hamiltonians produces a trivial action. The least demanding option for applying $H \in \mathbb{C G}-\mathbb{C G}^{\prime}$ relies then on the ability of fast-modulating $H$ according to the weak strength/fast switching scheme of [3].

Let $\mathcal{U}\left(\mathcal{C}_{J}\right)$ and $\mathcal{U}\left(\mathcal{D}_{J}\right)$ denote the subgroups of unitary transformations over the state space $\mathcal{C}_{J}$ and $\mathcal{D}_{J}$, respectively. Universality results can be established by observing that, by (2)-(3), $\left.\mathbb{C G}^{\prime}\right|_{\mathcal{C}_{J}} \simeq \operatorname{Mat}\left(n_{J} \times n_{J}, \mathbb{C}\right)=$ $\operatorname{End}\left(\mathcal{C}_{J}\right)$ and, similarly, $\left.\mathbb{C} \mathcal{G}\right|_{\mathcal{D}_{J}} \simeq \operatorname{Mat}\left(d_{J} \times d_{J}, \mathbb{C}\right)=$ $\operatorname{End}\left(\mathcal{D}_{J}\right)$ i.e., the elements of $\mathbb{C G}^{\prime}(\mathbb{C} \mathcal{G})$ restricted to the coding space span the whole operator algebra of the associated subsystem. Thus, by standard universality arguments [11], almost any pair of Hamiltonians $H_{i} \in \mathbb{C G}^{\prime}$ or $H_{i} \in \mathbb{C} \mathcal{G}, i=1,2$, is universal over $\mathcal{C}_{J}$ or $\mathcal{D}_{J}$, respectively. Similar existential results for control over commutant coordinates are formally derived in 10,7].

If $\mathbb{C G}$ is irreducible, the possibility to attain complete control over $\mathcal{H}_{S}$ [3] is directly found as a special case of the above results. When $\mathcal{G}$ acts reducibly, reachability of arbitrary states in $\mathcal{H}_{S}$ necessarily occurs through control operations that steer the system through different irreps of $\mathbb{C G}$ and $\mathbb{C G}^{\prime}$. The criteria for universality with no redundant encoding derived in [3] can then be regarded in terms of a symmetry mixing which arises from either combining commutant coordinates associated with different decouplers or from exploiting the action on both group and commutant coordinates of a single group $\mathcal{G}$.

It is worth stressing that complete controllability of noiseless subsystems does not by itself imply the potential of efficiently implementing a quantum network. This depends on the available physical Hamiltonians as well as on the details of the architecture by which subsystems are actually configured to encode and process information. We focus on quantum computation (QC).

Universal quantum computation.- Let $S$ be a quantum computer with $n$ qubits, $\mathcal{H}_{S} \simeq\left(\mathbb{C}^{2}\right)^{\otimes n}$. We consider henceforth a linear interaction Hamiltonian of the form

$$
H_{S B}=\sum_{a, i} \sigma_{a}^{(i)} \otimes B_{a}^{(i)},
$$

for suitable environment operators $B_{a}^{(i)}, a=x, y, z$, $i=1, \ldots, n$. Eq. (5) encompasses various models of interest where the error space is spanned by single-qubit Pauli operators. Notably, the two extreme situations of independent and collective decoherence correspond to error generators of the form $\left\{E_{\alpha}\right\}=\left\{\sigma_{a}^{(i)}\right\}, \operatorname{dim}(\mathcal{E})=3 n$, and $\left\{E_{\alpha}\right\}=\left\{\sum_{i} \sigma_{a}^{(i)}\right\}, \operatorname{dim}(\mathcal{E})=3$, respectively.

Example 1: The collective spin-flips decoupling group. Let us assume that $n$ is even and define $X_{j}=\sigma_{x}^{(j)}, Z_{j}=$ $\sigma_{z}^{(j)}$, with $Y_{j}=Z_{j} X_{j}=i \sigma_{y}^{(j)}$. The group of collective $\pi$-rotations is the set $\mathcal{G}=\left\{\mathbb{1}, \otimes_{i=1}^{n} X_{i}, \otimes_{i=1}^{n} Y_{i}, \otimes_{i=1}^{n} Z_{i}\right\}$. $\mathcal{G}$ is an Abelian subgroup of the Pauli group for $n$ qubits, with $k=2$ generators $\otimes_{i} X_{i}, \otimes_{i} Z_{i},|\mathcal{G}|=2^{k}=4$. Besides being identical with the stabilizer of distance-two $[n, n-2,2]$ error-correcting codes [12], $\mathcal{G}$ is also a subgroup of the full group of collective rotations that plays the role of a generalized stabilizer for noiseless codes within the collective decoherence model [13]. Decoupling with $\mathcal{G}$ is effective at suppressing any linear interaction of the form (5) since $\Pi_{\mathcal{G}}\left(\sigma_{a}^{(i)}\right)=0$. A single decoupling cycle is specified by a pulse sequence of the form $\left[\delta-\mathcal{P}_{x}-\delta-\mathcal{P}_{z}\right]^{2}, \delta=T_{c} / 4$ and $\mathcal{P}_{a}$ denoting a time delay and a collective $\pi$-pulse along the $\hat{a}$-axis respectively [3]. Since $\mathcal{G}$ is Abelian, $\mathcal{G}$ has $|\mathcal{G}|=4$ onedimensional irreps and the decomposition of $\mathcal{H}_{S}$ is identical to the decomposition according to joint eigenspaces $\mathcal{H}_{J}, J=1, \ldots, 2^{k}=4, \operatorname{dim}\left(\mathcal{H}_{J}\right)=n_{J}=2^{n-k}$. Encoding into commutant factors $\mathcal{C}_{J}$ is the only nontrivial option. Accordingly, each of the four (equivalent) joint $\mathcal{G}$-eigenspaces is able to encode $n-2$ logical qubits.

Control operations over each $2^{n-2}$-dim noiseless subspace can be implemented in the weak/slow fashion. Here is an explicit scheme for performing universal QC on encoded qubits. The key point is to look at the available operations in $\mathbb{C G}^{\prime}$, which is easily done by exploiting the isomorphism of $\mathcal{G}$ with the binary vector space $\mathcal{Z}_{2}^{2 n}$ along with standard results from stabilizer theory [12]. As a group, $\mathbb{C G}^{\prime}$ has a set of $2 n-2$ independent generators, two of which are also generators for $\mathcal{G}$. The $2(n-2)$ generators of $\mathbb{C G}^{\prime}-\mathcal{G}$ can be chosen among interactions of the form $X_{i} X_{j}, Z_{i} Z_{j}, i \neq j=1, \ldots, n$. These correspond to nontrivial encoded operations. For instance, the choice $\bar{X}_{j}=X_{1} X_{j+1}, \bar{Z}_{j}=Z_{j+1} Z_{n}, j=1, \ldots, n-2$, defines a set of $n-2$ logical qubits in terms of their encoded $\sigma_{x}$ and $\sigma_{z}$ observables $[14$. A universal set of quantum gates is generated by observing that $\mathbb{C G}^{\prime}$ also contains 
the Heisenberg couplings $\vec{\sigma}_{i} \cdot \vec{\sigma}_{j}=X_{i} X_{j}+Y_{i} Y_{j}+Z_{i} Z_{j}$ enabling one to implement swapping between any pair of encoded qubits i.e., $\vec{\sigma}_{\bar{i}} \cdot \vec{\sigma}_{\bar{j}}=\vec{\sigma}_{i+1} \cdot \vec{\sigma}_{j+1}$. Since the square-root-of-swap gate together with one-qubit gates are a universal set [15], we can noise-tolerantly perform universal $Q C$ on $n-2$ encoded qubits by slowly turning on and off two-body interactions in parallel with the decoupler.

Example 2: The symmetric decoupling group. Let $\mathcal{G}=\mathcal{S}_{n}$ be the natural representation of the permutation group on the $n$-fold tensor product space $\mathcal{H}_{S}$. In the presence of general linear interactions (5), decoupling according to $\mathcal{S}_{n}$ forces effective permutation symmetry, thereby simulating the collective decoherence model [0, 10,13]. Because $\Pi_{\mathcal{G}}(\mathcal{E})=\left\{\sum_{i} \sigma_{a}^{(i)}\right\} \subset \mathbb{C S}_{n}^{\prime}$, noiseless subsystems are only supported by group factors $\mathcal{D}_{J}$ carrying $\mathbb{C} \mathcal{S}_{n}$-irreps. By recalling that $\mathbb{C} \mathcal{S}_{n}^{\prime}$ is identical with the algebra of totally symmetric operators generated by the global $s u(2)$, the dimensions of such coding spaces can be calculated from the irrep multiplicities of angular momentum theory [9, ], 10]. Thus, $\operatorname{dim}\left(\mathcal{D}_{J}\right)=(2 J+1) n ! /[(n / 2+J+1) !(n / 2-J) !], J \in \mathbf{N} / 2$. An explicit scheme has been recently proposed for performing universal QC on logical qubits encoded in clusters of $n=4, J=0$ physical qubits $[13,16]$. The same construction applies in our setting, with the additional constraint that the exchange Hamiltonians required to implement universal gates should be fast-modulated at the same rate as the b.b. control within a cycle.

Example 3: The collective rotations decoupling group. Let $\mathcal{G}$ be the continuous group generated by the Lie algebra $\mathcal{L}=s u(2)$ of collective spin operators. Decoupling according to $\mathcal{G}$ can be achieved by performing the quantum operation (11) with respect to a suitable finiteorder symmetrizing group of unitaries $\mathcal{F}$, whose explicit form is given in [10]. Since $Z(\mathcal{G})=Z(\mathcal{L})=\mathbb{C} \mathcal{S}_{n}, \mathbb{C S}_{n^{-}}$ irreps emerge here as commutant factors, making this example the dual of the previous one. However, being $\Pi_{\mathcal{G}}(\mathcal{E})=\Pi_{\mathcal{F}}(\mathcal{E})=0$, noiseless subsystems can be supported now by both commutant factors, in which case $\operatorname{dim}\left(\mathcal{C}_{J}\right)=(2 J+1) n ! /[(n / 2+J+1) !(n / 2-J) !]$, or by group factors, for which $\operatorname{dim}\left(\mathcal{D}_{J}\right)=2 J+1$. In particular, if a $J=0$ four-qubits encoding in $\mathcal{C}_{J}$ is chosen as above, the scheme for universal QC proposed by [13] can be fully implemented according to weak/slow control.

Discussion.- We presented dynamical procedures for generating and controlling sectors of the state space of a generic open quantum system, which are (ideally) immune to environmental noise. In addition to substantially expanding the range of possibilities for using active decoupling methods, our analysis sheds light on the connections with passive error protection schemes, where the relevant degrees of freedom are decoupled from the noise-inducing interactions by virtue of preexisting symmetries. The presence of nontrivial symmetries is found to be at the root of both active and passive stabilization methods, thereby enabling the identification of common algebraic structures. In spite of the mathematical resemblance, however, the two strategies are physically very different. In particular, the limit of long reservoir correlation length, which underlies passive error prevention in the presence of collective noise [5], is replaced by the dynamical requirement of long reservoir correlation time in active decoupling, which explicitly relies on the nonMarkovian nature of quantum noise [2]. The combination of decoupling and coding procedures results in a scheme for performing universal quantum computation on noiseprotected subsystems which is highly appealing in terms of both the attainable encoding efficiency and the overall control resources. Even in the limit where environmental noise is fully tolerated, the scheme is not guaranteed to be robust against arbitrary errors due to imperfect control. The performance of decoupling in the presence of faulty control implementations along with the stability properties of the corresponding dynamically generated subsystems will be discussed in a forthcoming work.

L. V. is grateful to D. P. DiVincenzo for inspiring discussions on stabilizer codes. This work was supported by DARPA/ARO under the QUIC initiative. E. K. received support from the DOE, under contract W-7405-ENG-36, and from the NSA.

† vlorenza@mit.edu; knill@lanl.gov; slloyd@mit.edu

[1] L. Viola and S. Lloyd, Phys. Rev. A 58, 2733 (1998).

[2] L. Viola, E. Knill, and S. Lloyd, Phys. Rev. Lett. 82, 2417 (1999).

[3] L. Viola, S. Lloyd, and E. Knill, Phys. Rev. Lett. 83, 4888 (1999).

[4] E. Knill and R. Laflamme, Phys. Rev. A 55, 900 (1997), and references quoted therein.

[5] P. Zanardi and M. Rasetti, Phys. Rev. Lett. 79, 3306 (1997); L. M. Duan and G. C. Guo, Phys. Rev. Lett. 79, 1953 (1997); D. A. Lidar, I. L. Chuang, and K. B. Whaley, Phys. Rev. Lett. 81, 2594 (1998).

[6] E. Knill, R. Laflamme, and L. Viola, LANL e-print quantph/9908066, to appear in Phys. Rev. Lett.

[7] P. Zanardi, LANL e-print quant-ph/9910016.

[8] P. Zanardi, Phys. Lett. A 258, 77 (1999).

[9] J. F. Cornwell, Group Theory in Physics, Vol. I-III (Academic Press, London, 1984).

[10] P. Zanardi, Phys. Rev. A 60, R729 (1999).

[11] S. Lloyd, Phys. Rev. Lett. 75, 346 (1995).

[12] D. Gottesman, LANL e-print quant-ph/9705052.

[13] D. Bacon et al., LANL e-print quant-ph/9909058.

[14] In terms of basis states, the code subspace is spanned by the $\mathcal{G}$-invariant $n$-qubit cat-states $(|x\rangle+\mid$ NOT $x\rangle) / \sqrt{2}, x$ denoting an even-weigth binary string of length $n$.

[15] G. Burkard, D. Loss, and D. P. DiVincenzo, Phys. Rev. B 59, 2070 (1999).

[16] Similar constructive results can be established by starting from a more general subsystem encoding based on three physical qubits, L. Viola and E. Knill, in preparation. 